\title{
IntAct: a non-disruptive internal tagging strategy to study actin isoform organization and function
}

\author{
M.C. van Zwam ${ }^{1}$, W. Bosman ${ }^{1, *}$, W. van Straaten ${ }^{1, *}$, S. Weijers ${ }^{1}$, E. Seta ${ }^{1}$, B. Joosten ${ }^{1}$, and K. van den Dries ${ }^{1, \mathbb{}}$ \\ ${ }^{1}$ Department of Cell Biology, Radboud Institute for Molecular Life Sciences, Radboud University Medical Center, Nijmegen, The Netherlands \\ ${ }^{*}$ Contributed equally
}

\begin{abstract}
Actin plays a central role in many biological processes such as cell division, motility and contractility. In birds and mammals, actin has six, highly conserved isoforms, four of which are primarily present in muscles and two that are ubiquitously expressed across tissues. While each isoform has nonredundant biological functions, we currently lack the tools to investigate the molecular basis for isoform-specificity due to their high similarity and the limited possibilities to manipulate actin. To solve this technical challenge, we developed IntAct, an internally tagged actin system to study actin isoform organization in fixed and living cells. We performed a microscopy-based screen for 11 internal actin positions and identified one residue pair that allows for non-disruptive epitope tag integration. Using knockin cell lines with tags into the ubiquitously expressed $\beta$-actin, we demonstrate that IntAct actins are properly expressed and that their filament incorporation is indistinguishable from wildtype. We further show that IntAct actins can be visualized in living cells by exploiting the nanobody-targeted ALFA tag and that they keep their ability to interact with the actin-binding proteins profilin and cofilin. Lastly, we also introduced the tag in the ubiquitously expressed $\gamma$-actin and demonstrate that the differential localization observed for actin isoforms remains unaltered for IntAct actins. Together, our data demonstrate that IntAct is a promising tool to study actin isoform localization, dynamics and molecular interactions to finally enable the molecular characterization of actin isoforms in biological processes.
\end{abstract}

Actin isoforms | Cytoskeleton | Protein engineering | Microscopy

Correspondence: Koen.vandenDries@radboudumc.nl

\section{Introduction}

Actin plays a central role during fundamental biological processes including cell division, intracellular transport, motility and contractility. In birds and mammals, actin has six isoforms, also called isoactins, which are each encoded by different genes and expressed in a tissue and time-specific manner during development, homeostasis and pathology (13 ). Although exceptions exist, it is generally established that four isoactins are primarily expressed in muscle cells and two are ubiquitously expressed across tissues. The two ubiquitous isoactins, nonmuscle $\beta$ - and $\gamma$-actin, are extremely conserved and only differ four residues at their $\mathrm{N}$-terminus (4). Despite their extremely high similarity, $\beta$ - and $\gamma$-actin play a nonredundant role in many actin-controlled processes such as cell-cell junction formation (5), axon development (6), microtubule dynamics $(7,8)$, cell division $(9,10)$ and cell migration $(11,12)$. While isoactin-specific posttranslation mod- ification $(13,14)$, nucleation $(9,10)$ and translation speed $(14,15)$ have been demonstrated to play a role in the nonredundant role of $\beta$ - and $\gamma$-actin in cellular processes, many aspects of the molecular principles that govern the differential function of $\beta$ - and $\gamma$-actin are still unclear. This is mainly due to the limited possibilities to specifically probe actin isoforms for biochemical and cell biological assays.

Common tools to label the actin cytoskeleton such as phalloidin (16) in fixed cells or Lifeact (17), F-tractin (18), and UtrophinCH (19) in living cells do not discriminate between isoactins. Furthermore, C-terminal fusions of actin cannot be used to study actin biology since they only poorly assemble into filaments $(20,21)$. N-terminal fusions of actin have been used to study isoactin differences in cells $(15,22,23)$, but the reporter tags are known to significantly interfere with actin dynamics (17), nucleation (24) and molecular interactions $(17,24-26)$. Moreover, N-terminal fusion prevents isoactin-specific and nonspecific posttranslational modifications crucial for proper actin function such as arginylation $(13,14)$ and acetylation $(27,28)$. Attempts to tag yeast actin internally with a tetracysteine tag for contractile ring visualization were unsuccessful since none of the modified actins were integrated into the contractile ring filaments (29). An extended search for other internal sites that may be permissive for epitope tagging of actin has not been performed so far.

Here, we describe the development of a non-disruptive internal tagging strategy to study isoactin organization and function, which we call IntAct. For this, we first performed a microscopy-based screen for 11 internal actin positions and identified one residue pair that allows non-disruptive epitope tagging of actin. To prove its versatility and usability, we next engineerd CRISPR/Cas9-mediated knockin cell lines with various antibody- and nanobody-based epitope tags in the identified position and demonstrated that the internally tagged actins are properly expressed and that the integration into filaments is unperturbed. By performing immunofluorescence, pulldown experiments and live-cell imaging with the internally tagged actins, we show that IntAct can be used to study actin localization, molecular interactions and dynamics. Lastly, we show that isoactin-specific localization of actins appears unaffected, strongly suggesting that InAct can be provide unique insight into the isoactin-specific molecular principles that regulate cellular processes such as motility, division and intracellular transport. 
a

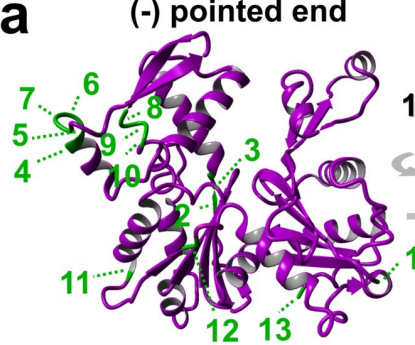

(+) barbed end
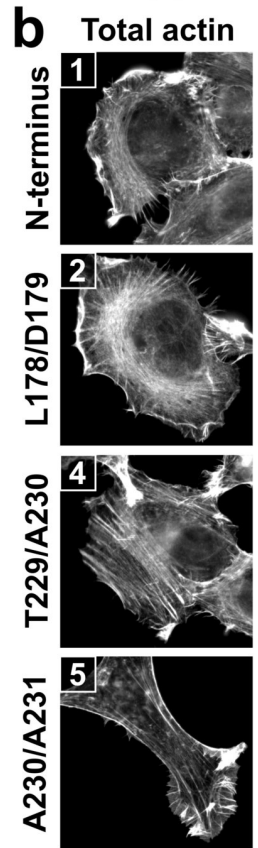

FLAG
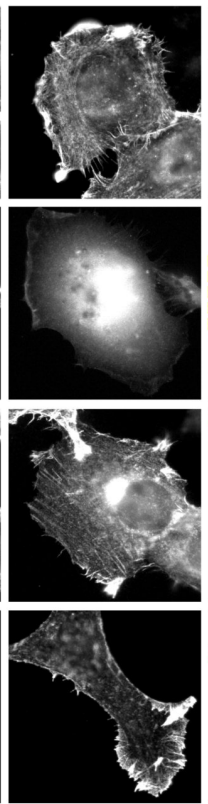

(-) pointed end

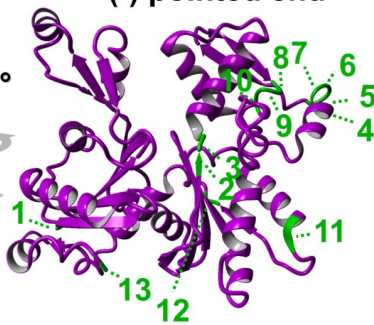

(+) barbed end
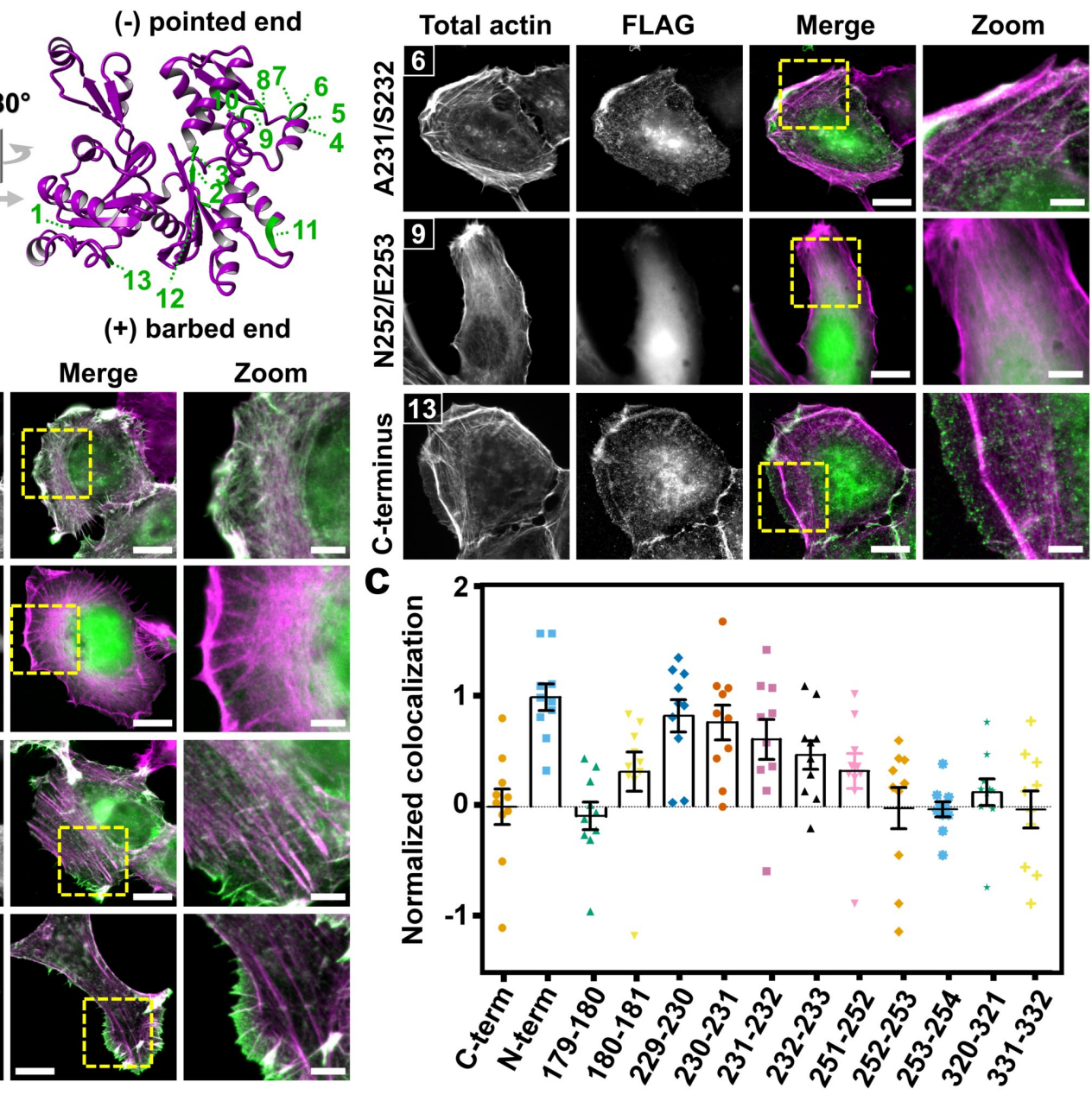

Fig. 1. Identification of actin T229/A230 as a permissive target site for epitope tag integration a, Crystal structure of uncomplexed globular actin (magenta ribbon, PBD accession number: $1 \mathrm{~J} 6 \mathrm{Z}(30)$ ) indicating the 11 internal target positions (green) as well as the $\mathrm{N}$ - and the C-terminus (1 and 13 , respectively). b, Representative widefield immunofluorescence images of total actin (magenta) and FLAG (green) in HT1080 cells that overexpress the tagged $\beta$-actin variants. Shown are 5 internally tagged variants and the $\mathrm{N}$ - and $\mathrm{C}$-terminally tagged $\beta$-actin. The remaining 6 internally tagged variants are shown in Suppl. Fig. S1. Scale bar: $15 \mu \mathrm{m}$. Scale bar zoom: $5 \mu \mathrm{m}$. c, Colocalization analysis of images shown in $\mathbf{b}$ showing the normalized Pearson's coefficient for each of the actin variants. Individual data points indicate single cells and in total, at least 10 cells from 2 independent experiments were included for the analysis.

\section{Results and Discussion}

T229/A230 actin residue pair is permissive for epitope tag insertion. To identify a permissive residue pair to internally tag the actin protein, we first performed a medium-scale screen tagging $\beta$-actin at eleven distinct residue pairs with a FLAG tag (Fig. 1a). These residue pairs were carefully selected, ensuring that at least one of the residues is part of an unstructured region (30) and that both residues were not involved in F-actin interactions (31). Furthermore, the first 40 amino acids were avoided since the coding mRNA for this region is involved in the different translation kinetics of actin isoforms (14). Eventually, two of the eleven selected positions were located in subdomain 3 , seven in subdomain 4 , and two were close to the ATP binding site. C- and N-terminally tagged $\beta$-actin were included in the screen as a negative and positive control for filament integration, respectively. We chose the FLAG tag as an epitope for our screen because of its frequent use, small size ( 8 amino acids, DYKDDDDK), and availability of a highly specific and well-characterized antibody (32).
To evaluate the integration of the tagged actins within the actin cytoskeleton, we overexpressed the 13 actin variants in human fibrosarcoma cells (HT1080, Fig. 1b-c, Fig. S1) and retinal pigment epithelium cells (RPE1, Fig. S2) and performed an immunofluorescence staining for the FLAG tag and phalloidin as a total F-actin marker (Fig. 1b, Fig. S1, Fig. S3a). Interestingly, by visual inspection, we observed that most of the internally tagged actins were diffusely present within the cytosol with two notable exceptions (T229/A230 and A230/A231). Of these two variants, A230/A231 only seemed to present a clear overlap with total actin at the cell periphery but the T229/A230 overlapped almost entirely with the total actin signal, similar to $\mathrm{N}$ terminally tagged actin. To quantitatively assess the colocalization of the tagged actin variants with the F-actin cytoskeleton, we performed a Pearson coefficient analysis (Fig. 1c, Fig. S1b). As expected, N-terminally tagged actin showed a high degree of colocalization, and C-terminally tagged actin showed almost no colocalization. We therefore performed a unity-based correction, adjusting the Pearson coefficient of 


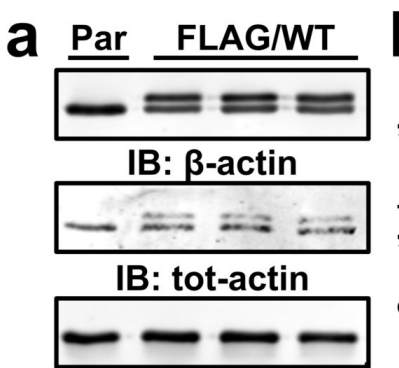

IB: Y-actin
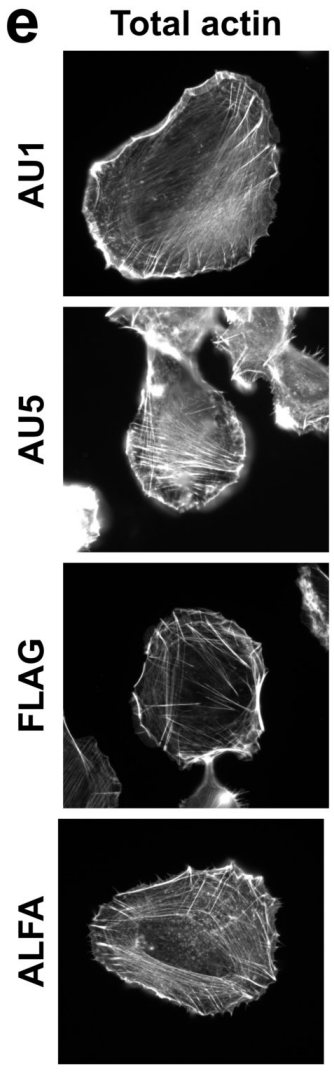

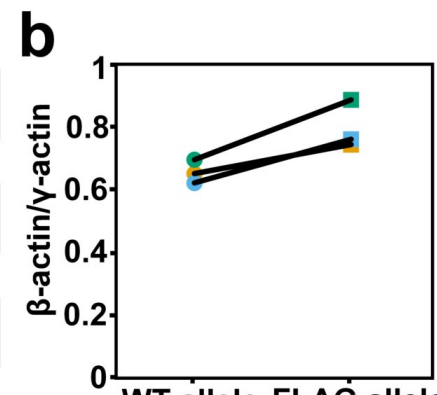

WT allele FLAG allele
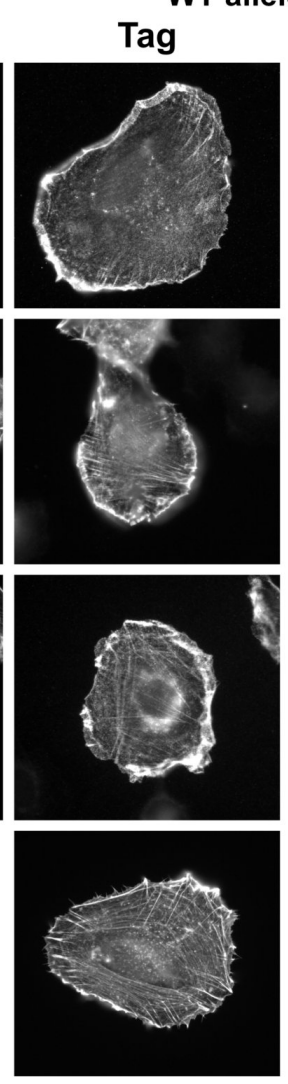

C

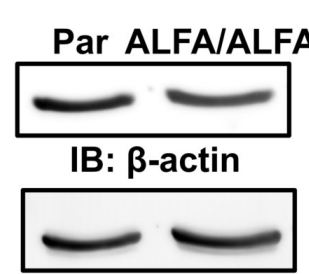

IB: Tubulin
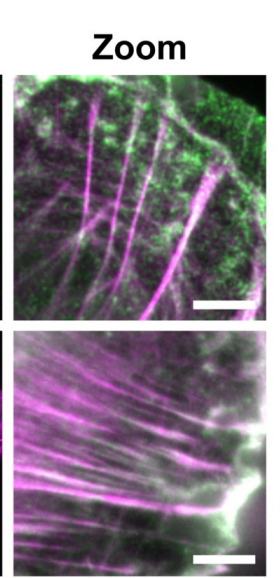
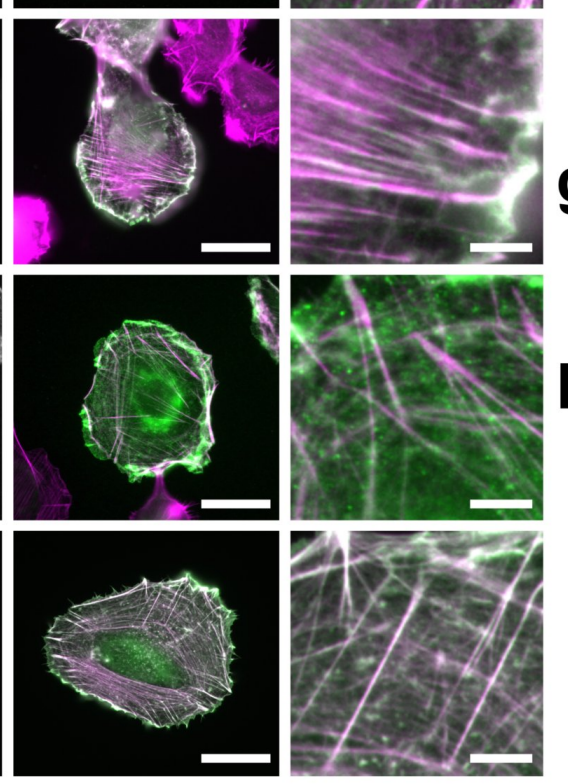
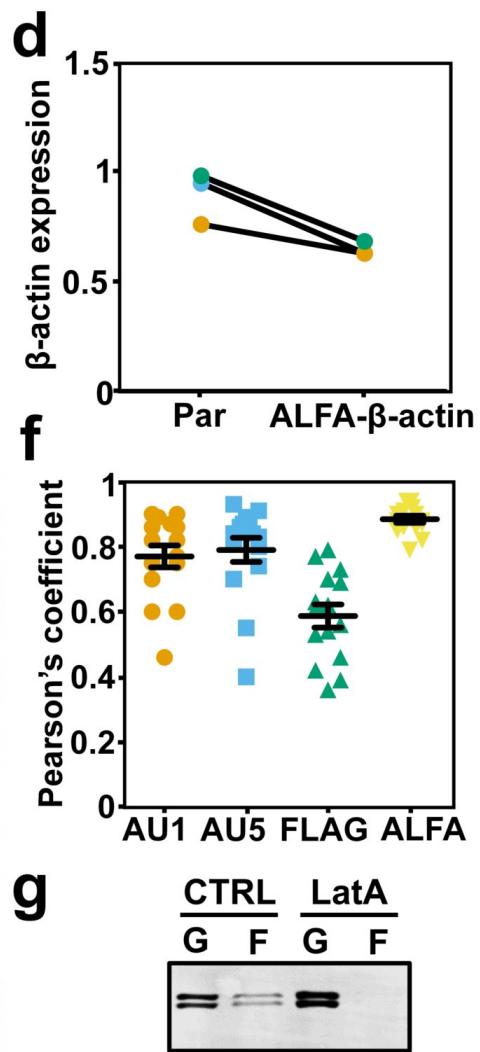

h

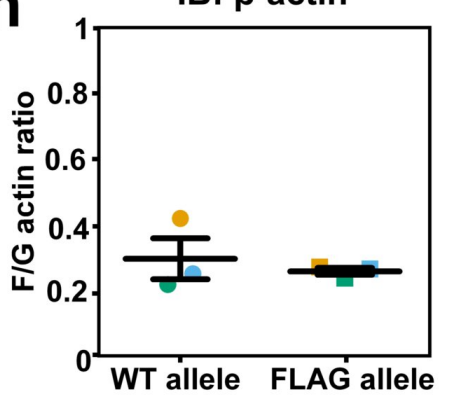

Fig. 2. Actin functionality unperturbed by T229/A230 epitope integration a, Western blot of $\beta$-actin, total actin and $\gamma$-actin in parental HT1080 (Par) and heterozygous FLAG- $\beta$-actin HT1080 cells (FLAG/WT). b, Quantification of $\beta$-actin protein expressed by the WT allele and the FLAG allele as shown in a and normalized to $\gamma$-actin. $\mathbf{c}$, Representative western blot showing $\beta$-actin expression in parental HT1080 (Par) and homozygous ALFA- $\beta$-actin HT1080 cells (ALFA/ALFA). d, Quantification of the $\beta$-actin expression from the western blot shown in c and normalized to tubulin. e, Representative widefield images from cells that have a CRISPR/Cas9-mediated knockin of AU1, AU5, FLAG or ALFA tag in $\beta$-actin. Cells were labeled for phalloidin and an antibody/nanobody against the respective tag to visualize total actin (magenta) and the tagged $\beta$-actin (green). Scale bar: $15 \mu \mathrm{m}$. Scale bar zoom: $5 \mu \mathrm{m}$. f, Colocalization analysis of the microscopy results in e showing the Pearson's coefficient for each of the internally tagged actins. Individual data points indicate single cells and in total, at least 10 cells from 2 independent experiments were included in the analysis. $\mathrm{g}$, Representative western blot of G-actin and F-actin fraction in heterozygous FLAG- $\beta$-actin HT1080 cells that were left untreated or treated with latrunculin A. $\mathbf{h}$, Quantification of the F-/G-actin ratio for $\beta$-actin expressed by the WT allele and FLAG allele from the western blots shown in $\mathbf{g}$.

the $\mathrm{C}$ - and $\mathrm{N}$-terminues to zero and one, respectively, and normalized the other values within this window. While most of the internally tagged actin variants showed little to no colocalization, the T229/A230 displayed a high Pearson coefficient in both HT1080 and RPE1 cells (raw $\mathrm{R}^{2}=0.68$ and 0.75 , respectively), confirming our initial observation and indicating that this internally tagged actin is well integrated into the F-actin cytoskeleton. These results strongly suggest that the T229/A230 residue pair in $\beta$-actin is permissive for epitope tag insertion without affecting the ability of actin to be incorporated within filaments.
T229/A230 epitope tag insertion does not impair actin expression or assembly into filaments. To investigate the versatility and usability of the T229/A230 residue pair for epitope tag insertion, we applied CRISPR/Cas9-mediated homology-directed repair (HDR) to genetically introduce various tags into this position at the genomic locus of $\beta$-actin. We included the antibody-based epitope tags FLAG, AU1 (DTYRYI) and AU5 (TDFYLK) as well as the recently developed nanobody $(\mathrm{Nb})$-based ALFA tag (PSRLEEELRRRLTEP, (33)). Of note, we introduced the AU5 tag without the starting threonine (DFYLK), since this residue is redundant with the T229 of actin. Eventually, we obtained cells with 
one properly modified allele for AU1, AU5 and FLAG, and homozygous knockin cells for the ALFA tag as confirmed by Sanger sequencing (Fig. S3).

After generation of the knockin cells, we evaluated the actin protein expression since it has been shown that a heterozygous knockin of green fluorescent protein (GFP) into the genomic locus of $\beta$-actin leads to a dramatic decrease of protein expression from the modified allele (25). For this, we used heterozygous FLAG- $\beta$-actin cells since the FLAG tag causes a gel shift on western blot, allowing a direct assessment of tagged and wildtype actin expression in the same cells (Fig. 2a-b). Quantification of the western blots demonstrated that the amount of FLAG- $\beta$-actin was slightly higher compared to wildtype indicating that the cells did not downregulate the expression of $\beta$-actin from the knockin allele. We further evaluated the protein expression in the homozygous ALFA- $\beta$-actin cells and this showed that the total amount of actin was about 20 percent lower in the ALFA- $\beta$-actin cells compared to parental HT1080 cells (Fig. 2c-d). To exclude that this minor decrease compromised global actin regulation in the ALFA- $\beta$-actin cells, we evaluated the expression of $\gamma$ actin and $\alpha$-smooth muscle actin ( $\alpha$-SMA) as a genetic loss of $\beta$-actin has been shown to induce the expression of these isoforms (34). Importantly, we neither observed differences in $\gamma$-actin expression or an induction of $\alpha$-SMA in the ALFA$\beta$-actin cells (Fig. S4), strongly suggesting that global actin regulation is not perturbed by the genetic tagging of $\beta$-actin.

Next, we assessed the incorporation of the tagged actins into the cytoskeleton by performing immunofluorescence labeling followed by widefield microscopy (Fig. 2e). Pearson colocalization analysis demonstrated that all the knockin tagged actins have a strong overlap with the total actin, indicating that they are well incorporated in the F-actin cytoskeleton (Fig. 2f). Since the ALFA tag allows intracellular detection of the tagged actins in living cells, we also performed live-cell imaging with the ALFA- $\beta$-actin cells. For this, we overexpressed ALFA-Nb-mScarlet in the ALFA- $\beta$ actin cells and evaluated its colocalization with F-actin by cotransfecting Lifeact-GFP and determining the Pearson correlation coefficient at multiple time points (Fig. S5, Suppl. Movie 1). This demonstrated that, also in living cells, there is a very high correlation $\left(R^{2}=>0.8\right)$ between the fluorescence intensity of the tagged actins and the Lifeact-GFP signal (Fig. S4), further supporting the notion that the internally tagged $\beta$-actin is properly assembled into actin filaments.

To corroborate our microscopy results, we sought to biochemically determine the F/G-actin ratio of the tagged and wildtype actin and for this, we again used the heterozygous FLAG- $\beta$-actin cells. F-actin was purified using high speed centrifugation and cells stimulated with Latrunculin A to disrupt F-actin were included as a negative control (Fig. 2g). The results from these exeriments demonstrated that the F/G-actin ratio for FLAG- $\beta$-actin was indistinguishable from wildtype actin (Fig. 2h), indicating that FLAG- $\beta$-actin was normally integrated into actin filaments.

Together, these results in fixed and living cells with multiple epitope tags suggest that the T229/A230 residue pair
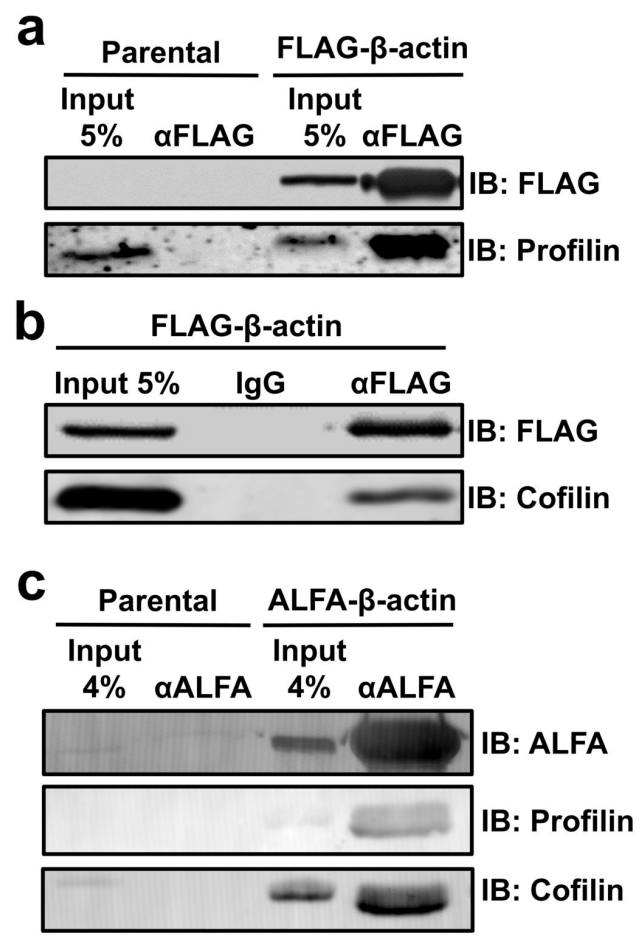

Fig. 3. FLAG- and ALFA- $\beta$-actin interact with profilin and cofilin a, Representative western blot showing the co-immunopreciptation of FLAG- $\beta$-actin and profilin using an anti-FLAG antibody in the FLAG- $\beta$-actin HT1080 cells. Coimmunoprecipitation performed on parental HT1080 was included as a control. b, Representative western blot showing the co-immunopreciptation of FLAG- $\beta$-actin and cofilin using an anti-FLAG antibody. IgG was included as a negative control. c, Representative western blot showing the co-immunopreciptation of ALFA- $\beta$-actin and profilin and cofilin using an anti-ALFA nanobody in the ALFA- $\beta$-actin HT1080 cells. Co-immunoprecipitation performed on parental HT1080 was included as a control.

in actin is a versatile position for epitope tagging with only a minor impact on actin expression and no measurable influence on the ability of actin to integrate into filaments.

Internally tagged actins interact with cofilin and profilin. To study the molecular interactions of the internally tagged $\beta$-actin, we performed a co-immunoprecipitation assay and western blot analysis using FLAG- $\beta$-actin and ALFA- $\beta$-actin. Since co-immunoprecipitation of actin only allows the investigation of monomeric G-actin interactors, we evaluated the binding of the well-established G-actin binding proteins cofilin and profilin. Importantly, we first concluded that both FLAG- and ALFA- $\beta$-actin could be immunoprecipitated from the lysates (Fig. 3a-c), confirming the availability of the epitope tags under native conditions. Moreover, we could demonstrate that FLAG- $\beta$-actin (Fig. 3a-b) as well as ALFA- $\beta$-actin (Fig. 3c) still associate with cofilin and profilin, indicating that the internally tagged actins maintain their ability to bind to these crucial actin regulatiors.

Actin polymerization and cell function is unperturbed in ALFA- $\beta$-actin cells. Fluorescent fusions of actin are known to affect the polymerization dynamics at the cell front, likely due to the large fluorescent reporter tag (17). To evaluate whether actin polymerization dynamics is unperturbed by 
bioRxiv preprint doi: https://doi.org/10.1101/2021.10.25.465733; this version posted October 28, 2021. The copyright holder for this preprint (which was not certified by peer review) is the author/funder, who has granted bioRxiv a license to display the preprint in perpetuity. It is made available under aCC-BY-ND 4.0 International license.
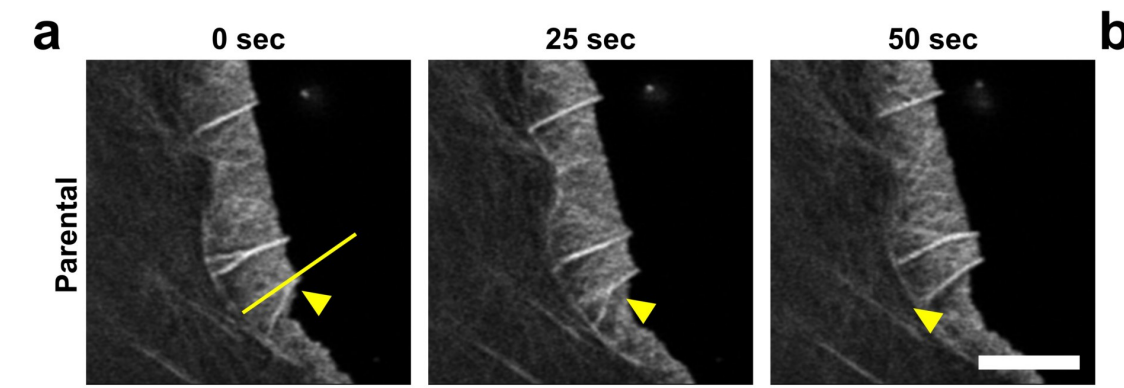

b
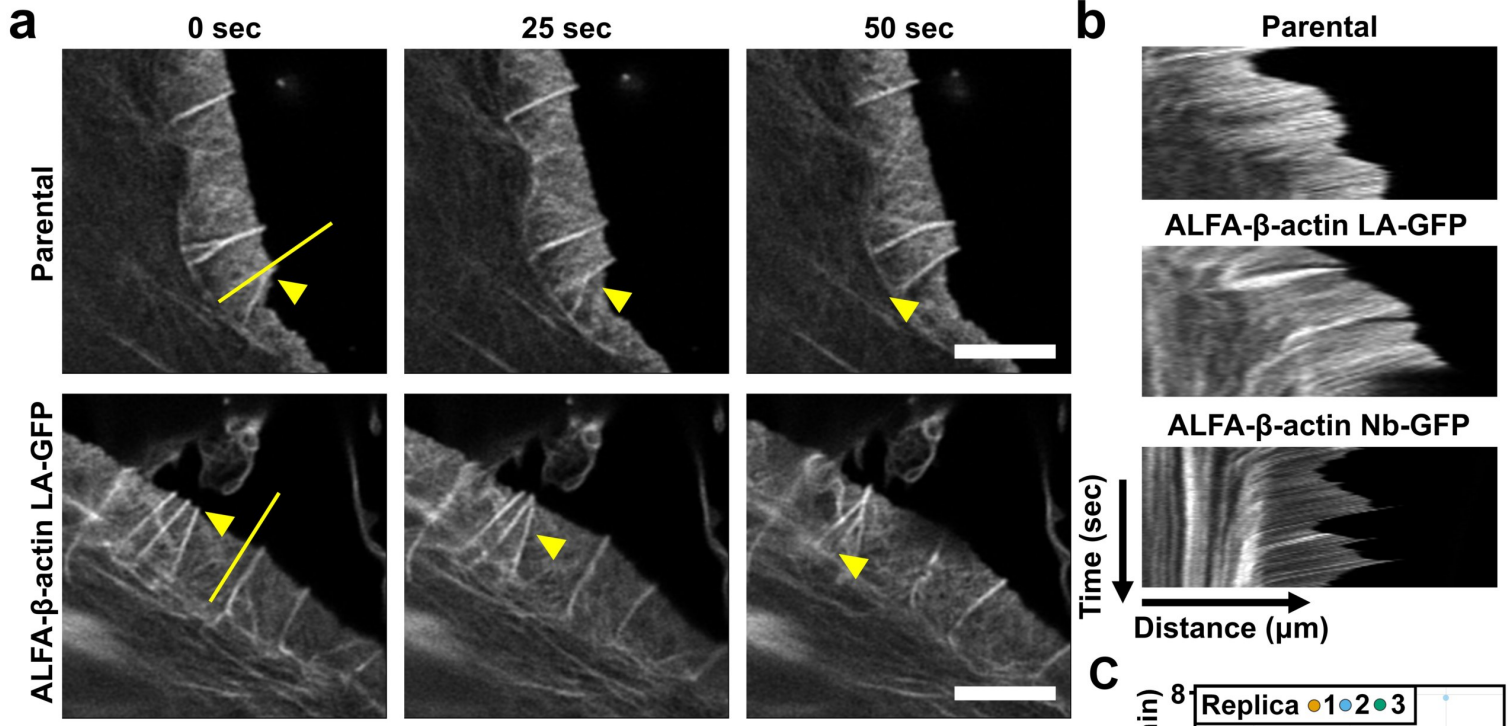

ALFA- $\beta$-actin Nb-GFP
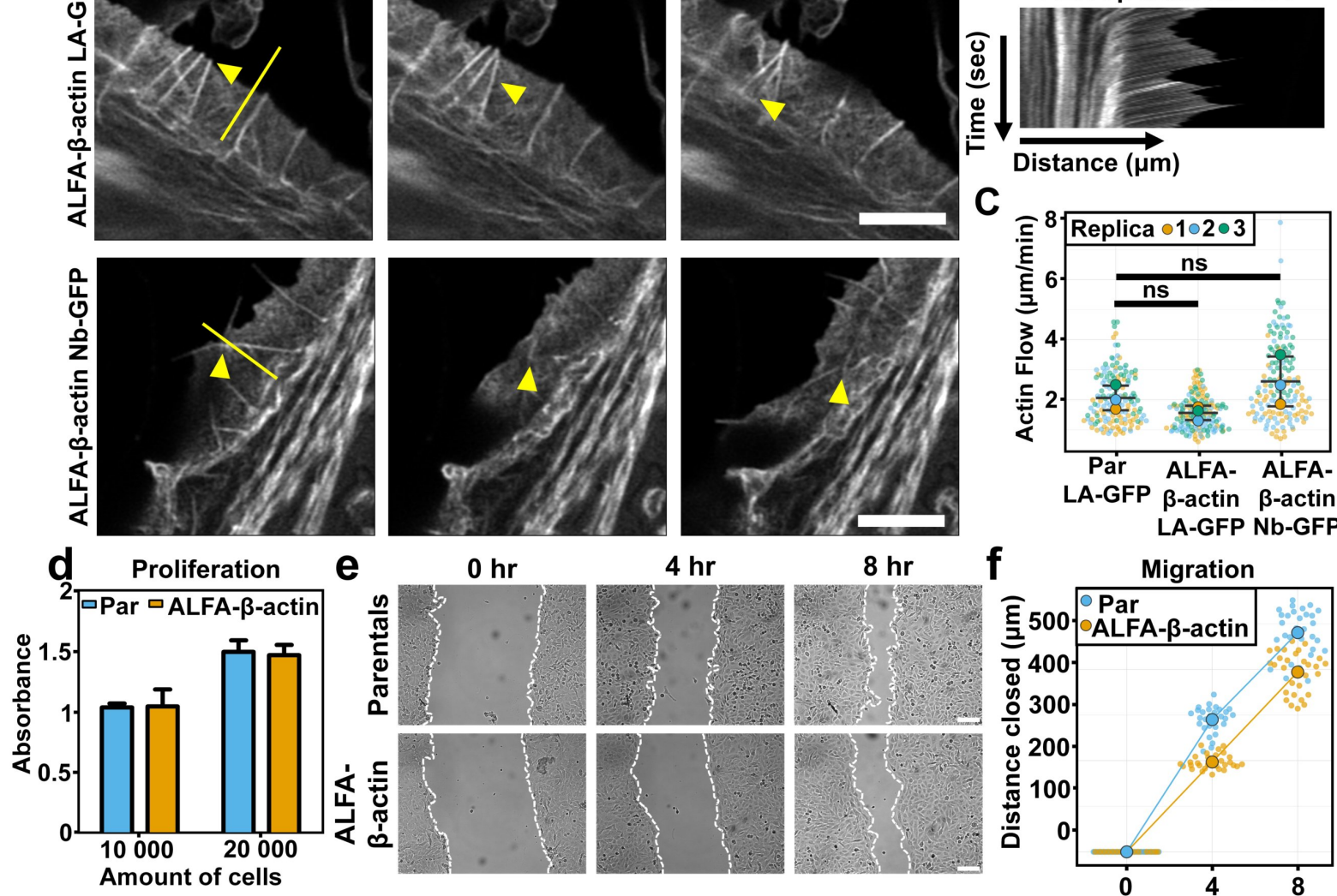

$\mathbf{O ~ h r}$

$4 \mathrm{hr}$

$8 \mathrm{hr}$

C
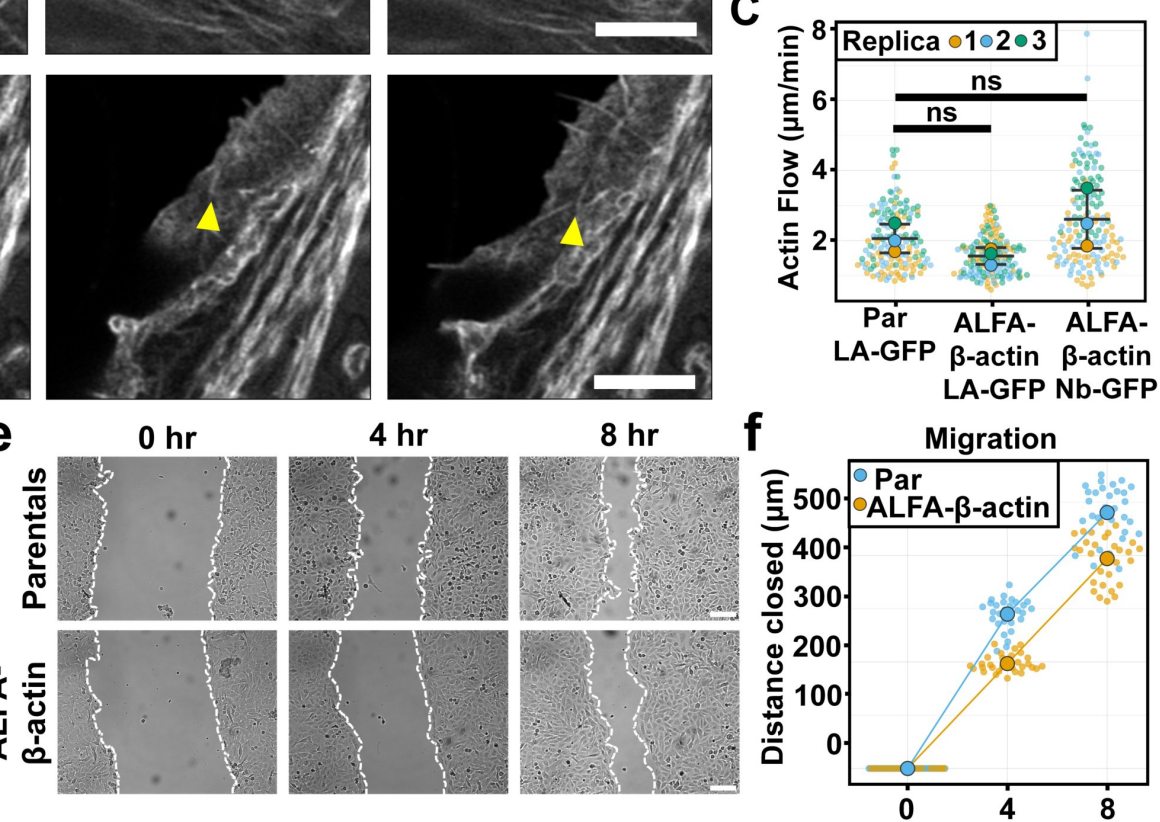

Fig. 4. Actin treadmilling and cell proliferation and migration are not affected by $\beta$-actin internal tagging a, Representative airyscan images of $H T 1080$ parental cells transfected with Lifeact-GFP (LA-GFP), HT1080 ALFA- $\beta$-actin cells transfected with Lifeact-GFP and HT1080 ALFA- $\beta$-actin cells transfected with ALFA-Nb-GFP. Shown are three stills and the yellow triangles indicate actin features that display rearward treadmilling. Yellow line indicates the position of kymographs shown in $\mathbf{b}$. The full movies are available as Suppl. Movies 2-4. Scale bar: $4 \mu \mathrm{m}$. b, Representative kymographs of parental, ALFA $\beta$ actin-LA-GFP and ALFA- $\beta$-actin-Nb-GFP as indicated by the yellow line in a. c, Quantification of the actin flow $(\mu \mathrm{m} / \mathrm{min})$ in parentals-LA-GFP, ALFA- $\beta$-actin-LA-GFP, ALFA- $\beta$ actin-Nb-GFP. Large datapoints represent the average for each experiment and the small datapoints represent individual kymographs. The error bars show the median and the 25th and 75th percentile. Statistical analysis was performed using unpaired Welch's t-test. Parentals-LA-GFP vs ALFA- $\beta$ actin-LA-GFP $P=0.16$. Parentals-LA-GFP vs ALFA- $\beta$-actin-Nb-GFP $P=0.38$. d, Quantification of an MTT proliferation assay performed on parental and ALFA- $\beta$-actin HT1080 cells. Bars and error indicate the average and standard error of the mean of 3 experiments. e, Representative widefield images of parental and ALFA- $\beta$-actin HT1080 cells at time point $0 \mathrm{hr}, 4 \mathrm{hr}$ and $8 \mathrm{hr}$ after scratch induction. Scale bar: $30 \mu \mathrm{m}$. f, Quantification of the scratch assay shown in e indicating the distance closed in $\mu \mathrm{m}$ over time in parental (Par) and ALFA- $\beta$-actin HT1080 cells. Large data points represent the mean of 2 experiments and the small data points represent the quantification of the individual images. 15 images per condition were acquired per experiment.

introducing the internal tag at position T229/A230, we determined the actin treadmilling speed at lamellipodia using live cell imaging (Fig 4a). For this, we transfected ALFA$\beta$-actin cells with Lifeact-GFP or ALFA-Nb-GFP and performed time lapse imaging with Airyscan super-resolution microscopy. Parental cells transfected with Lifeact-GFP were included as a control since the expression of Lifeact has been demonstrated to not affect the actin treadmilling speed at the cell front (17). Importantly, we observed actin treadmilling at lamellipodia in all of the conditions indicating no gross defects in the formation of these structures by the internal ALFA tag (Suppl. Movie S2-4). Moreover, by quantitative analysis of the kymographs from the time-lapse videos, we demonstrate that there are no significant differences in the treadmilling speed at lamellipodia between any of the investigated conditions (Fig. $\mathbf{4 b}$-c). These results strongly suggest that actin polymerization dynamics is not disturbed by the internal tag in $\beta$-actin.

To demonstrate that the internal tag does not influence cellular processes that are crucially dependent on proper 
actin function, we evaluated the ability of ALFA- $\beta$-actin cells to proliferate and migrate as compared to parental HT1080 cells. To assess cell proliferation, we performed an MTT assay and observed no differences in the the proliferation rate between ALFA- $\beta$-actin and parental HT1080 cells (Fig. 4d). To assess cell migration, we performed a scratch assay. Althoug the migration rate of the ALFA- $\beta$-actin cells was slightly lower compared to parental HT1080 cells, they were still able to close the scratch and we only observed a different migration speed in the first 4 hrs (Fig. 4e-f).

Together, these results indicate that the molecular dynamics as well as major actin-dependent cellular functions are largely unaffected when actin is tagged at postion T229/A230.

Tagged $\beta$ - and $\gamma$-actin recapitulate differential isoform distribution. So far, our results strongly suggest that the T229/A230 position in actin is a permissive position for nondisruptive epitope tag integration. We term this internal tagging strategy "IntAct" and propose that it can be used to study the molecular principles of actin isoform specificity in biological processes. To indeed demonstrate that the tagged actins recapitulate the behaviour of wildtype isoforms, we also engineered an HT1080 IntAct knockin cells with an ALFA tag in the genomic locus of $\gamma$-actin. We then evaluated the localization of the actin isoforms in the parental as well as the IntAct $\beta$ - and $\gamma$-actin HT1080 cells, since it has been shown before that these isoform display a differential cellular distribution $(12,35)$. As expected, in the parental HT1080 cells, $\beta$-actin is enriched at the cell periphery and $\gamma$-actin is equally distributed throughout the entire cell (Fig. 5a). More importantly, when comparing the localization of tagged $\beta$ and $\gamma$-actin to total actin in the IntAct cells, we observed that the tagged $\beta$ - and $\gamma$-actin recapitulate the differential isoform distribution (Fig. 5a). This strongly suggests that isoformspecific properties remain preserved after internal tagging at position T229/A230.

Collectively, the results presented in this manuscript from fixed and living cells demonstrate that IntAct is a versatile genetic system to study the localization, dynamics and molecular interactions of actin. At this point, we can only speculate as to why the T229/A230 position seems permissive for manipulation. The T229/A230 residue pair is located in subdomain 4 and is part of a region that has been termed the V-stretch due to the high structural variation that this region exhibits in molecular dynamics simulations of F-actin (36). To the best of our knowledge, the V-stretch has no explicitly described interactions with actin-binding proteins and, unlike other variable regions such as the D-loop, is not involved in interactions between monomers in actin filaments (37). Interestingly, an alanine mutagensis scan of the entire $\beta$-actin protein demonstrated that the V-stretch demonstrates high structural plasticity since the alanine mutants covering this region were not impaired in their folding capacity or their binding to the actin-binding proteins DNAse I, adseverin, Thymosin $\beta 4$ and CAP (38). Moroever, the T229/A230 position is very close to S232/S233, a residue pair that was included in a previous screen to internally tag actin in yeast
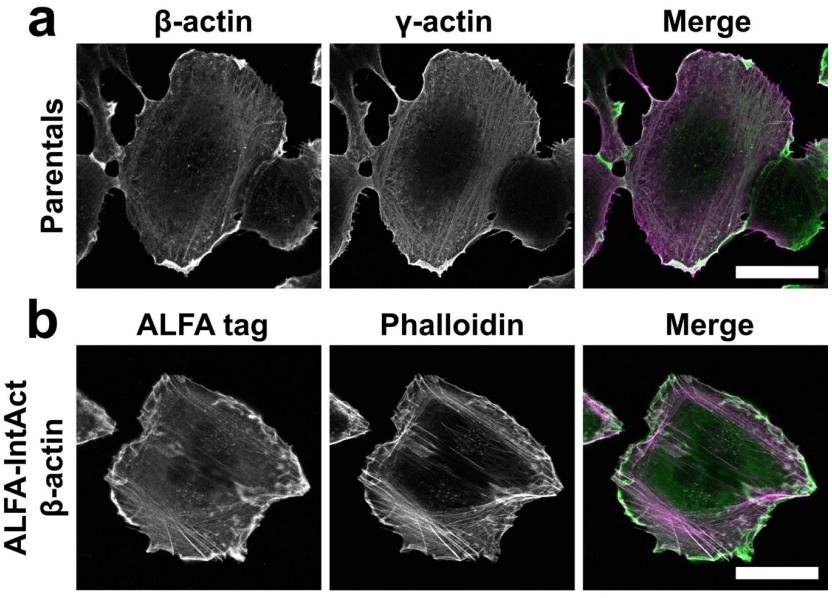

Phalloidin

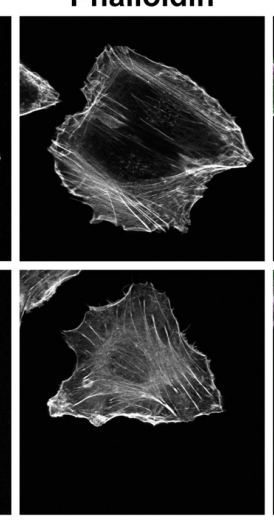

Merge

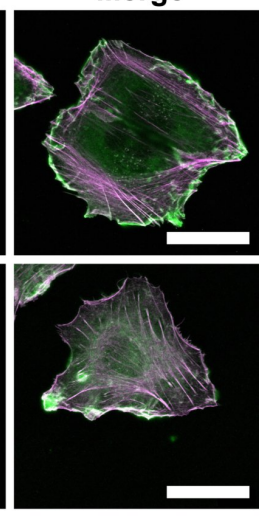

Fig. 5. IntAct $\beta$ - and $\gamma$-actin recapitule differential distribution of actin isoforms a, Representative Airyscan images of parental HT1080 stained for $\beta$-actin (green) and $\gamma$-actin (magenta). b, Representative Airyscan images of ALFA- $\beta$-actin and ALFA- $\gamma$-actin HT1080 cells stained with phalloidin and an anti-ALFA nanobody to visualize total actin (magenta) and the internally tagged actins (green), respectively. Scale bar: $15 \mu \mathrm{m}$

and which was the only variant that assembled into yeast actin cables, albeit only weakly (29). In our screen, we also included the S232/S233 as well as the A230/A231 and A231/S232 positions but these internally tagged variant were not as well assembled into filaments as the T229/A230 suggesting very specific structural requirements for actin internal tagging. Importantly, based on the extremely high similarity between isoactins, we predicted that the internal position could be used for both nonmuscle $\beta$ - and $\gamma$-actin. We indeed demonstrate that $\beta$ - and $\gamma$-actin can be tagged at this position and that the localization of the IntAct isoactins is similar the wildtype, strongly suggesting that IntAct is a promising tool for the investigation of the molecular principles behind the nonredundant roles of isoactins in cellular processes such as migration and division.

\section{Experimental Procedures}

Cell Culture. HT1080 fibrosarcoma cells were used for the overexpression of internally tagged actins and to generate the internally tagged cell lines. Cells were cultured in $1 \mathrm{x}$ DMEM + 4.5 g/L D-Glucose, NEAA (Gibco, Lot\#2246377) and supplemented with $10 \%$ ( $\mathrm{vol} / \mathrm{vol}$ ) fetal bovine serum (FBS), 1X Glutamax (Gibco, 2063631), 1 mM Sodium Pyruvate (Gibco, 2010382) and 0.5X Antibiotic-Antimycotic (Gibco, 15240-062). RPE1 cells were used for the overexpression of internally tagged actins. RPE1 cells were cultered in advanced DMEM/F-12 + non-essential amino acids $+110 \mathrm{mg} / \mathrm{L}$ Sodium Puruvate (Gibco, Lot\#12634010) supplemented with $10 \%$ (vol/vol) fetal bovine serum (FBS) and $1 \mathrm{X}$ 
Glutamax (Gibco, 2063631). All cell lines were cultured and kept at $37^{\circ} \mathrm{C}$ with $5 \% \mathrm{CO} 2$.

Antibodies and reagents. The following primary antibodies were used (dilution is indicated for immunofluorescence unless stated otherwise): anti- $\beta$-actin (\#MCA5775GA, Bio-Rad Laboratories, 1:200 dilution, 1:2000 for western blot), anti- $\gamma$-actin (\#MCA5776GA, Bio-Rad Laboratories, 1:200 dilution, 1:2000 for western blot), anti-Flag (\#F1804-1MG, Sigma Aldrich, 1:100), anti-AU1 (\#NB600452, Novus biologicals, 1:100), anti-AU5 (\#NB600-461, Novus biologicals), anti-profilin (\#3246, Cell Signaling), anti-cofilin (\#5175P, Cell Signalling). Secondary antibodies conjugated to Alexa647, Alexa555, Alexa568, or Alexa 488 were used (Life Technologies, 1:400 dilution). Actin was stained with Alexa-568-conjugated phalloidin (Life Technologies, 1:200 dilution), ALFA-tag was stained with antiALFA-atto488 conjugate. D-Biotin was purchased from sigma Aldrich (\#B4639).

Generation of overexpression constructs. All overexpression constructs with the internally tagged actins were generated by Gibson assembly (New England Biolabs). Briefly, two PCRs were performed per construct to generate a DNA fragment before the tag and a fragment after the tag. Primers used for the PCR reactions are given in the Supplementary Materials. Both fragments contained the DNA coding for the tag which functions as an overlapping sequence in the Gibson assembly reaction. The pcDNA3.1 vector backbone was linearized using HindIII and NheI and 100ng of vector was used in every Gibson assembly. PCR fragments were added in a 1:6 vector:insert molar ratio and all assembly reactions were incubated for 50 degrees for approximately 2 hrs. Half the product was transformed into Top10 competent bacteria and clones were screened for the correct vectors.

Generation of knockin cell lines. gRNAs and HDR templates used for the generation of FLAG-, AU1-, AU5t- and ALFA-knock-in cells are given in the Supplementary Materials. Lipofectamine 2000 (ThermoFisher, ref. 11668027) was used to transfect the HT1080 cells. To increase the efficacy of the knockin approach we applied a coselection procedure using ouabain as described previously (39). The gRNA and HDR template for mutating the ATP1A1, which leads to ouabain resistance, are given in the supplementary materials. Flow cytometery for the respective tags was performed two weeks after the initial transfection to determine the number of positive cells. Subsequently, treatment with $0.75 \mu \mathrm{M}$ ouabain was started and after two weeks, single clones were generated from the selected cells. Positive clones were selected based on intracellular FACS staining and were further used for immunofluorescence and western blot.

Immunofluorescence. All steps were performed at room temperature. Cells were seeded on coverslips and fixed using 4\% PFA for 10 min. Permeabilization was performed with $0.1 \%$ Triton $\mathrm{X}-100$ for 5 min. After washing with $1 \mathrm{x}$ PBS, the samples were blocked with $20 \mathrm{mM}$ PBS+glycin. Primary Ab incubation was performed for $1 \mathrm{hr}$. Subsequently, samples were washed $3 x$ with $1 x$ PBS and incubated with the appropriate secondary $\mathrm{Ab}$ for $1 \mathrm{hr}$ in the dark. The samples were then washed $2 \mathrm{x}$ with $1 \mathrm{x}$ PBS and $1 \mathrm{x}$ with MiliQ. After these washing steps the samples were sealed in Mowiol and dried overnight.

Imaging. Imaging was performed on a confocal Zeiss LSM 900 and Leica DMI6000 epifluorescence microscope. Imaging data on LSM 900 was aquired using a x63 1.4 NA oil objective. Alexa488 was ecxited at $517 \mathrm{~nm}$ and emmission was detected between 400-560. Alexa555 was excited at $568 \mathrm{~nm}$ and emission was detected between 560$700 \mathrm{~nm}$. Alexa568 was excited at $603 \mathrm{~nm}$ and emmission was detected between 560-700 nm. Raw images were reconstructed using the Zeiss Zen 3.1 blue edition software. Imaging data on Leica DMI6000 was aquired with an HC PL APO $\times 63 / 1.40-0.60$ oil objective and a metal halide lamp. Alexa488 was excited through a BP $470 / 40 \mathrm{~nm}$ and emission was detected through a BP 525/50 nm. Alexa568 was excited through a BP 546/12 nm and emission was detected through a BP $605 / 75 \mathrm{~nm}$. Raw images were reconstructed using the LAS AF 3.2 software. Images were analyzed using ImageJ. Pearson coefficient was calculated using the ImageJ tool Coloc2 (PSF: 3, Costes randomisations 10).

Live-cell imaging. Parental and/or ALFA- $\beta$-actin HT1080 cells were seeded in Wilco dishes. The next day, cells were transfected with Lifeact-GFP, Nb-ALFA-GFP or Nb-ALFA-mScarlet together with LifeAct using Lipofectamine 2000 (Invitrogen, lot\#1854327). DMEM was replaced by imaging medium (HBSS, $\mathrm{Ca} / \mathrm{Mg}, 5 \%$ FCS, $1 \mathrm{M}$ HEPES) and incubated for approximately $10 \mathrm{~min}$ prior to imaging. Live cell imaging was performed using an LSM 880, airyscan data was acquired using a x63 1.4 NA oil objective. Emission light was collected using a BP 495-550 + LP 570 for LifeAct-GFP and Nb-GFP. Emission light was collected using a BP 420-480/BP 495-550 for LifeAct-GFP together with Nb-mScarlet. Raw images were reconstructed using the Zeiss Zen $2.1 \mathrm{Sp} 1$ software. Time series were collected with a frame interval of $5 \mathrm{sec}$ for actin treadmilling and $15 \mathrm{sec}$ for colocalization of LifeAct with Nb-mScarlet. The movies were analyzed using ImageJ and the Pearson correlation coefficient was calculated using the ImageJ tool Coloc2 (PSF: 3, Costes randomisations 10).

Western blot. For western blot, analysis samples were mixed with 2x Laemmli (0.5 M Tris pH 6.8, 10x SDS, Glycerol, bromphenol blue, mercaptoethanol). After denaturing the samples, they were loaded onto $10 \%$ or $15 \%$ SDS-PAGE gels for separation. Separation was accomplished by running for \pm 2 hours (hr) at $100 \mathrm{~V}$ in 1x Running buffer (10XTBS, 10x SDS). Proteins were transferred to PVDF membranes for $\pm 1 \mathrm{hr}$ at $100 \mathrm{~V}$ in Transfer buffer (10XTBS, MeOH). Membranes were blocked in 5\% milk in TBST (1x TBS, Tween20) and incubated with primary antibodies overnight at $4{ }^{\circ} \mathrm{C}$ while turning. After washing with $1 \mathrm{x}$ TBST, membranes were incubated in the dark for $1 \mathrm{hr}$ with secondary antibodies while spinning. Washing with $1 \mathrm{x}$ TBST was repeated and subsequently, the protein bands were visualized using Typhoon FLA 7000 (GE Healthcare). ImageJ was used to analyze the protein bands. 
F-/G-actin ratio. Heterozygous FLAG- $\beta$-actin knockin clones were seeded and the next day washed in ice-cold PBS and lysed on ice for 10 minutes with F-actin stabilization buffer (0.1 M PIPES pH 6.9, 30\% glycerol, 5\% DMSO, 1 mM MgSO4, 1 mM EGTA, 1\% Triton X-100, 1 mM ATP, protease inhibitor cocktail (Sigma, 11697498001)). The cells were harvested and spun down for 10 minutes at 1,000 $\mathrm{g}$ and $4^{\circ} \mathrm{C}$. The supernatant was collected and spun down at 16,000 $\mathrm{g}$ for 75 minutes at $4^{\circ} \mathrm{C}$ to separate the G- and F-actin fractions. The supernatant, containing G-actin, was collected and the pellet, containing F-actin, was solubilized in depolymerization buffer (0.1 M PIPES pH 6.9, $1 \mathrm{mM} \mathrm{MgSO} 4,10 \mathrm{mM}$ $\mathrm{CaCl} 2,5 \mu \mathrm{M}$ cytochalasin D, $1 \%$ SDS). For the negative control, cells were treated with $1 \mu \mathrm{M}$ Latrunculin A 30 minutes before lysis to disrupt the F-actin fraction. The F-/G-ratio was determined by western blot analysis.

ALFA tag co-immunoprecipitation. Cells were seeded and the following day, the cells were washed five times with ice-cold PBS. Cells were lysed with ice-cold lysis buffer (10 $\mathrm{mM}$ Tris, $150 \mathrm{mM} \mathrm{NaCl}, 2 \mathrm{mM} \mathrm{MgCl} 2,2 \mathrm{mM} \mathrm{CaCl} 2,1 \%$ Brij-97), supplemented with 1x protease inhibitor cocktail (Roche, 11697498001) and 0.1 mM PMSF (Sigma-Aldrich, P7626-5G). Cell lysates were centrifugation at $16,000 \mathrm{~g}$ for 60 minutes at $4^{\circ} \mathrm{C}$. For ALFA tag pulldowns, ALFA-Selector ST beads (NanoTag biotechnologies, N1510) were washed twice with lysis buffer. For input $4 \%$ or $5 \%$ of the clarified lysate was collected as positive control and diluted in $2 \mathrm{x}$ Laemmli buffer. The rest of the sample was combined with the beads and incubated for 1 hour at $4^{\circ} \mathrm{C}$ with rotation. After enrichment, the beads were pelleted by centrifugation for 1 minute at $1,000 \mathrm{~g}$, and the supernatant was collected as negative control. The beads were washed five times with lysis buffer and incubated for 20 minutes with $2 \mathrm{x}$ Laemmli buffer supplemented $0.2 \mu \mathrm{M}$ elution peptide (NanoTag biotechnologies, N1520-L) at RT with subtle shaking. The samples were centrifuged for 1 minute at 3,000 $\mathrm{g}$ and the supernatant collected as elute sample.

FLAG co-immunoprecipitation. The day before starting the co-immunoprecipitation protocol, BSA- and IgG-coated dynabeads for pre-clearing were prepared by mixing dynabeads slurry ( $40 \mu \mathrm{l}$ per sample) with $500 \mu \mathrm{l} \mathrm{PBS} / 3 \%$ BSA or $500 \mu \mathrm{l}$ PBS with $2.5 \mu \mathrm{g}$ mouse IgG1 (BioLegend, 400102) respectively. Similarly, BSA-coated dynabeads for the IP itself were prepared by mixing $60 \mu \mathrm{l}$ dynabeads slurry per sample with $500 \mu \mathrm{l}$ PBS/3\% BSA. FLAG knock-in clones were seeded and when the cells reached full confluency, they were washed once with cold PBS and lysed for 15 minutes at $4^{\circ} \mathrm{C}$ with $1 \mathrm{ml}$ lysis buffer (1\% Brij-97, $10 \mathrm{mM}$ Tris- $\mathrm{HCl} \mathrm{pH} \mathrm{7.5,}$ $150 \mathrm{mM} \mathrm{NaCl}, 2 \mathrm{mM} \mathrm{MgCl} 2,2 \mathrm{mM} \mathrm{CaCl}$, protease inhibitor cocktail (Sigma, 11697498001)). Cell lysates were collected by scraping and spun down at $16,000 \mathrm{~g}$ and $4{ }^{\circ} \mathrm{C}$ for 75 minutes to remove F-actin. In the meantime, the pre-clear beads were washed once with lysis buffer (with or without 1 $\mathrm{mM}$ ATP) and resuspended in a total of $40 \mu \mathrm{l}$ per sample. The supernatant was pre-cleared for 1 hour at $4^{\circ} \mathrm{C}$ while shaking, with both the BSA- and IgG-coated beads. After preclearing, the beads were removed and $40 \mu$ of the supernatant was collected as input. The rest of the sample was split into two parts, which was supplemented with lysis buffer to a volume of $2 \mathrm{ml} .6 \mu \mathrm{g}$ of either mouse anti-FLAG (Sigma, F1804) or mouse IgG1 (BioLegend, 400102) was added and samples were incubated for 1 hour at $4^{\circ} \mathrm{C}$ under rotation. Meanwhile, the IP beads were washed with lysis buffer (with or without $1 \mathrm{mM}$ ATP) and resuspended in a total of $60 \mu \mathrm{l}$ per sample. The beads were added and the samples were incubated for an additional 2 hours. After washing five times with washing buffer (1\% Brij-97, $10 \mathrm{mM}$ Tris- $\mathrm{HCl} \mathrm{pH} 7.5,150 \mathrm{mM} \mathrm{NaCl}$, $2 \mathrm{mM} \mathrm{MgCl} 2,2 \mathrm{mM} \mathrm{CaCl} 2,1 \mathrm{mM}$ PMSF), the beads were eluted in $100 \mathrm{mM}$ glycine $\mathrm{pH} 3.0$ for 5 minutes while rotating. The samples were neutralized by adding $1 / 10$ th volume of $1 \mathrm{M}$ Tris- $\mathrm{HCl} \mathrm{pH} 8.5$ and loaded on 15\% SDS-PAGE gels for western blot analysis.

Scratch assay. Cells were seeded to $100 \%$ confluency and a scratch was made with a $200 \mu \mathrm{l}$ tip from the top to the bottom of the well. After scratching, the cells were washed $1 \mathrm{x}$ with PBS and fresh media was added to the cells. The same position was imaged with a Leica DMI6000 epifluorescence microscopeafter $0 \mathrm{hr}, 4 \mathrm{hr}$ and $8 \mathrm{hr}$ and the images were analyzed using ImageJ.

MTT proliferation assay. Cells (10.000 or 20.000) were seeded in a 96-well plate. After 16 hrs of incubation, media was replaced by media containing the tetrazolium dye MTT $(0.45 \mathrm{mg} / \mathrm{ml})$. After $2 \mathrm{hrs}$ of MTT incubation at $37 \mathrm{C}$ in the $\mathrm{CO} 2$ incubator, $150 \mu \mathrm{l}$ DMSO was added to the cells and incubater for $10 \mathrm{~min}$ on an orbital shaker until the crystals are dissolved. The absorbance at $560 \mathrm{~nm}$ was read by a microplate reader (iMark microplate absorbance reader, Bio$\mathrm{Rad})$.

Statistics. The type of statistical test, $n$ values, and $P$ values are all listed in the figure legends or in the figures. All statistical analyses were performed using Graph Pad Prism or Microsoft Excel, and significance was determined using a 95\% confidence interval.

Data availability. All primary data supporting the conclusions made are available from the authors on request.

\section{ACKNOWLEDGEMENTS}

We are indebted to NanoTag Biotechnologies GmbH, Göttingen, Germany for providing us with the ALFA nanobody expression construct. The authors further thank the Radboud Technology Center Microscopy of the Radboudumc for the use of their microscopy facilities. This work was financially supported by intramural funding of the Radboudumc and by an NWO KLEIN grant (OCENW.KLEIN.494) awarded to K.D..

\section{AUTHOR CONTRIBUTIONS}

All authors performed experiments. E.S. generated the overexpression constructs. S.W., W.B. and W.S. generated the HT1080 knockin cells. W.B and M.C.Z. performed the co-immunoprecipitation experiments. M.C.Z. performed the overexpression experiments, live-cell imaging, proliferation and migration experiments. K.D. and M.C.Z. carried out data analysis, prepared all the figures and wrote the manuscript. K.D. conceived and supervised the study.

\section{Bibliography}

1. L. R. Andrade. Evidence for changes in beta- and gamma-actin proportions during inner ear hair cell life. Cytoskeleton (Hoboken), 72(6):282-91, 2015.

2. K. M. McHugh, K. Crawford, and J. L. Lessard. A comprehensive analysis of the developmental and tissue-specific expression of the isoactin multigene family in the rat. Dev Biol, 148(2):442-58, 1991.

3. D. Tondeleir, D. Vandamme, J. Vandekerckhove, C. Ampe, and A. Lambrechts. Actin isoform expression patterns during mammalian development and in pathology: insights from mouse models. Cell Motil Cytoskeleton, 66(10):798-815, 2009. 
bioRxiv preprint doi: https://doi.org/10.1101/2021.10.25.465733; this version posted October 28, 2021. The copyright holder for this preprint (which was not certified by peer review) is the author/funder, who has granted bioRxiv a license to display the preprint in perpetuity. It is made available under aCC-BY-ND 4.0 International license.

4. B. J. Perrin and J. M. Ervasti. The actin gene family: function follows isoform. Cytoskeleton (Hoboken), 67(10):630-4, 2010.

5. S. Baranwal, N. G. Naydenov, G. Harris, V. Dugina, K. G. Morgan, C. Chaponnier, and A. I. Ivanov. Nonredundant roles of cytoplasmic beta- and gamma-actin isoforms in regulation of epithelial apical junctions. Mol Biol Cell, 23(18):3542-53, 2012.

6. M. Moradi, R. Sivadasan, L. Saal, P. Luningschror, B. Dombert, R. J. Rathod, D. C. Dieterich, R. Blum, and M. Sendtner. Differential roles of alpha-, beta-, and gamma-actin in axon growth and collateral branch formation in motoneurons. J Cell Biol, 216(3):793-814, 2017.

7. V. Dugina, I. Alieva, N. Khromova, I. Kireev, P. W. Gunning, and P. Kopnin. Interaction of microtubules with the actin cytoskeleton via cross-talk of eb1-containing +tips and gammaactin in epithelial cells. Oncotarget, 7(45):72699-72715, 2016.

8. S. T. Po'uha, S. Honore, D. Braguer, and M. Kavallaris. Partial depletion of gamma-actin suppresses microtubule dynamics. Cytoskeleton (Hoboken), 70(3):148-60, 2013.

9. A. Chen, P. D. Arora, C. A. McCulloch, and A. Wilde. Cytokinesis requires localized betaactin filament production by an actin isoform specific nucleator. Nat Commun, 8(1):1530 2017.

10. A. Chen, L. Ulloa Severino, T. C. Panagiotou, T. F. Moraes, D. A. Yuen, B. D. Lavoie, and A. Wilde. Inhibition of polar actin assembly by astral microtubules is required for cytokinesis. Nat Commun, 12(1):2409, 2021.

11. V. Dugina, I. Zwaenepoel, G. Gabbiani, S. Clement, and C. Chaponnier. Beta and gammacytoplasmic actins display distinct distribution and functional diversity. J Cell Sci, 122(Pt 16):2980-8, 2009.

12. E. Pasquier, M. P. Tuset, S. Sinnappan, M. Carnell, A. Macmillan, and M. Kavallaris. gammaactin plays a key role in endothelial cell motility and neovessel maintenance. Vasc Cell, 7 : 2, 2015.

13. M. Karakozova, M. Kozak, C. C. Wong, A. O. Bailey, 3rd Yates, J. R., A. Mogilner, H. Zebroski, and A. Kashina. Arginylation of beta-actin regulates actin cytoskeleton and cell motility. Science, 313(5784):192-6, 2006.

14. F. Zhang, S. Saha, S. A. Shabalina, and A. Kashina. Differential arginylation of actin isoforms is regulated by coding sequence-dependent degradation. Science, 329(5998):15347, 2010.

15. P. Vedula, S. Kurosaka, B. MacTaggart, Q. Ni, G. Papoian, Y. Jiang, D. W. Dong, and A. Kashina. Different translation dynamics of beta- and gamma-actin regulates cell migration. Elife, 10, 2021.

16. J. A. Cooper. Effects of cytochalasin and phalloidin on actin. J Cell Biol, 105(4):1473-8, 1987.

17. J. Riedl, A. H. Crevenna, K. Kessenbrock, J. H. Yu, D. Neukirchen, M. Bista, F. Bradke, D. Jenne, T. A. Holak, Z. Werb, M. Sixt, and R. Wedlich-Soldner. Lifeact: a versatile marker to visualize f-actin. Nat Methods, 5(7):605-7, 2008.

18. M. J. Schell, C. Erneux, and R. F. Irvine. Inositol 1,4,5-trisphosphate 3-kinase a associates with f-actin and dendritic spines via its $n$ terminus. J Biol Chem, 276(40):37537-46, 2001.

19. B. M. Burkel, G. von Dassow, and W. M. Bement. Versatile fluorescent probes for actin filaments based on the actin-binding domain of utrophin. Cell Motil Cytoskeleton, 64(11): 822-32, 2007.

20. V. Brault, U. Sauder, M. C. Reedy, U. Aebi, and C. A. Schoenenberger. Differential epitope tagging of actin in transformed drosophila produces distinct effects on myofibril assembly and function of the indirect flight muscle. Mol Biol Cell, 10(1):135-49, 1999.

21. H. Rommelaere, D. Waterschoot, K. Neirynck, J. Vandekerckhove, and C. Ampe. A method for rapidly screening functionality of actin mutants and tagged actins. Biol Proced Online, 6 : 235-249, 2004.

22. A. Simiczyjew, A. J. Mazur, E. Dratkiewicz, and D. Nowak. Involvement of beta- and gammaactin isoforms in actin cytoskeleton organization and migration abilities of bleb-forming human colon cancer cells. PLoS One, 12(3):e0173709, 2017.

23. A. Simiczyjew, A. J. Mazur, A. Popow-Wozniak, M. Malicka-Blaszkiewicz, and D. Nowak. Effect of overexpression of beta- and gamma-actin isoforms on actin cytoskeleton organization and migration of human colon cancer cells. Histochem Cell Biol, 142(3):307-22, 2014.

24. J. Q. Wu and T. D. Pollard. Counting cytokinesis proteins globally and locally in fission yeast. Science, 310(5746):310-4, 2005

25. B. Roberts, A. Haupt, A. Tucker, T. Grancharova, J. Arakaki, M. A. Fuqua, A. Nelson, C. Hookway, S. A. Ludmann, I. A. Mueller, R. Yang, R. Horwitz, S. M. Rafelski, and R. N. Gunawardane. Systematic gene tagging using crispr/cas9 in human stem cells to illuminate cell organization. Mol Biol Cell, 28(21):2854-2874, 2017.

26. M. Westphal, A. Jungbluth, M. Heidecker, B. Muhlbauer, C. Heizer, J. M. Schwartz, G. Marriott, and G. Gerisch. Microfilament dynamics during cell movement and chemotaxis monitored using a gfp-actin fusion protein. Curr Biol, 7(3):176-83, 1997.

27. A. Drazic, H. Aksnes, M. Marie, M. Boczkowska, S. Varland, E. Timmerman, H. Foyn, N. Glomnes, G. Rebowski, F. Impens, K. Gevaert, R. Dominguez, and T. Arnesen. Naa80 is actin's n-terminal acetyltransferase and regulates cytoskeleton assembly and cell motility. Proc Natl Acad Sci U S A, 115(17):4399-4404, 2018.

28. M. Goris, R. S. Magin, H. Foyn, L. M. Myklebust, S. Varland, R. Ree, A. Drazic, P. Bhambra, S. I. Stove, M. Baumann, B. E. Haug, R. Marmorstein, and T. Arnesen. Structural determinants and cellular environment define processed actin as the sole substrate of the n-terminal acetyltransferase naa80. Proc Natl Acad Sci U S A, 115(17):4405-4410, 2018.

29. Q. Chen, S. Nag, and T. D. Pollard. Formins filter modified actin subunits during processive elongation. J Struct Biol, 177(1):32-9, 2012.

30. L. R. Otterbein, P. Graceffa, and R. Dominguez. The crystal structure of uncomplexed actin in the adp state. Science, 293(5530):708-11, 2001.

31. M. Boczkowska, Z. Yurtsever, G. Rebowski, M. J. Eck, and R. Dominguez. Crystal structure of leiomodin 2 in complex with actin: A structural and functional reexamination. Biophys $\mathrm{J}$, 113(4):889-899, 2017.

32. A. Einhauer and A. Jungbauer. Affinity of the monoclonal antibody $\mathrm{m} 1$ directed against the flag peptide. J Chromatogr A, 921(1):25-30, 2001.

33. H. Gotzke, M. Kilisch, M. Martinez-Carranza, S. Sograte-Idrissi, A. Rajavel, T. Schlichthaerle, N. Engels, R. Jungmann, P. Stenmark, F. Opazo, and S. Frey. The alfa-tag is a highly versatile tool for nanobody-based bioscience applications. Nat Commun, 10(1):
4403, 2019.

34. P. Vedula and A. Kashina. The makings of the 'actin code': regulation of actin's biological function at the amino acid and nucleotide level. J Cell Sci, 131(9), 2018.

35. K. van den Dries, L. Nahidiazar, J. A. Slotman, M. B. M. Meddens, E. Pandzic, B. Joosten, M. Ansems, J. Schouwstra, A. Meijer, R. Steen, M. Wijers, J. Fransen, A. B. Houtsmuller, P. W. Wiseman, K. Jalink, and A. Cambi. Modular actin nano-architecture enables podosome protrusion and mechanosensing. Nat Commun, 10(1):5171, 2019.

36. T. Splettstoesser, K. C. Holmes, F. Noe, and J. C. Smith. Structural modeling and molecular dynamics simulation of the actin filament. Proteins, 79(7):2033-43, 2011.

37. D. S. Kudryashov and E. Reisler. Atp and adp actin states. Biopolymers, 99(4):245-56, 2013.

38. H. Rommelaere, D. Waterschoot, K. Neirynck, J. Vandekerckhove, and C. Ampe. Structural plasticity of functional actin: pictures of actin binding protein and polymer interfaces. Structure, 11(10):1279-89, 2003.

39. D. Agudelo, A. Duringer, L. Bozoyan, C. C. Huard, S. Carter, J. Loehr, D. Synodinou, M. Drouin, J. Salsman, G. Dellaire, J. Laganiere, and Y. Doyon. Marker-free coselection for crispr-driven genome editing in human cells. Nat Methods, 14(6):615-620, 2017. 